\title{
Revisión sistemática: La dermatoscopía es más precisa que el examen clínico en el diagnóstico de melanomas
}

Is Dermoscopy (Epiluminescence Microscopy) Useful for the diagnosis of Melanoma?. Bafounta ML, Beauchet A, Aegerter P et al. Arch Dermatol 2001;137:1343-1350.

\section{Objetivo}

Determinar la precisión de la dermastoscopia, en el diagnóstico de melanoma frente al mero examen clínico, por personal experimentado.

\section{Fuente y Selección de Datos}

Los autores buscaron trabajos en distintas bases de datos (MEDLINE, EMBASE, PASCAL-BIOMED y BIUM) hasta el 31 de mayo de 2000. Los estudios fueron seleccionados por los siguientes criterios: 1 - que incluyan melanomas y lesiones bien descriptas dentro de un espectro de lesiones que comúnmente se confunden con melanomas, 2- que las comparaciones de test diagnósticos sean ciegas (dermoscopía y diagnóstico clínico) y que se utilice la histología como criterio estándar y 3- que se puedan calcular valores de sensibilidad y especificidad y de cocientes de probabilidad positivos y negativos*. Solo 8 de 672 estudios detectados cumplieron estos criterios de validez.

\section{Resultados Principales}

Los estudios seleccionados abarcaron a 328 melanomas (la mayoría de un espesor menor a $0.76 \mathrm{~mm}$.) y a 1865 lesiones de piel melanociticas benignas.
Tabla: Resultados principales

\begin{tabular}{l|c|c|c|c}
\hline Test & $\begin{array}{c}\text { Sensibilidad } \\
\text { (rango) }\end{array}$ & $\begin{array}{c}\text { Especificidad } \\
\text { (rango) }\end{array}$ & $\begin{array}{c}\text { Coeficiente de } \\
\text { Probabilidad } \\
\text { Positivo (IC 95\%) }\end{array}$ & $\begin{array}{c}\text { Coeficiente de } \\
\text { Probabilidad } \\
\text { Negativo (IC 95\%) }\end{array}$ \\
\hline Diagnóstico clínico & 50 a 94\% & 55 a 94\% & $3,7(2,8$ a 5,3) & $0,27(0,19-0.36)$ \\
\hline Dermatoscopía & 75 a 96\% & 79 a 98\% & $9(5,6$ a 19) & $0,11(0,05$ a 0,18) \\
\hline
\end{tabular}

Ver glosario para las características operativas de pruebas diagnósticas.

La dermatoscopía mostró un poder de discriminación y de sensibilidad mayor que el examen clínico a través de comparar sus curvas $\mathrm{ROC}^{*}$, y esto fue estadísticamente significativo. No se pudo encontrar una relación entre la sensibilidad diagnóstica de la dermatoscopía con el numero de lesiones analizadas, el porcentaje de melanomas, el tipo de dermatoscopio o los criterios dermatoscópicos utilizados.

\section{Conclusión}

Para personal experimentado, la dermatoscopía es mas precisa que el examen clínico para el diagnóstico de melanoma, frente a una lesión pigmentada de piel.

\section{Comentario}

La dermatoscopía es una técnica in vivo, no invasiva que ofrece una nueva visión de la morfología clínica de las estructuras pigmentadas de la epidermis, de la unión dermoepidérmica y de la dermis papilar.

Esta técnica mejora la sensibilidad y la especificidad para el diagnóstico clínico de tumores pigmentados. La importancia de lograr este diagnóstico se basa en la necesidad de una detección precoz del melanoma, pero también la dermatoscopía facilita el reconocimiento de lesiones cutáneas pigmentadas no melanomas como el epitelioma basocelular pigmentado, angiomas y queratosis seborreicas. Esto permite muchas veces evitar la exéresis inútil de nevos benignos.

Hay que tener en cuenta que todos los trabajos 1,2 muestran un au- mento de la sensibilidad en el diagnóstico de melanoma en relación al examen clínico, en dermatólogos entrenados, que trabajan dentro de centros especializados con experiencia en dematoscopía. Considerando la evidencia disponib le, sería recomendable que los dermatólogos realizaran un esfuerzo para incorporar esta herramienta tan útil.

Conclusiones del comentador: Los estudios disponibles en la literatura concluyen que la dermatoscopía realizada por dermatólogos es una técnica más precisa que el examen clínico, de utilidad tanto para el diagnóstico precoz como para reducir las intervenciones en lesiones benignas. 